\title{
Synaptic modifications depend on synapse location and activity: a biophysical model of STDP
}

\author{
A. Saudargiene ${ }^{\mathrm{a}},{ }^{\mathrm{b}}$, B. Porr ${ }^{\mathrm{a}}$ and F. Wörgötter ${ }^{\mathrm{a}}$ \\ ${ }^{a}$ Department of Psychology, University of Stirling, Stirling, Scotland, UK \\ ${ }^{\mathrm{b}}$ Department of Informatics, Vytautas Magnus University, Kaunas, Lithuania
}

In spike-timing-dependent plasticity (STDP) the synapses are potentiated or depressed depending on the temporal order and temporal difference of the pre- and postsynaptic signals. We present a biophysical model of STDP which assumes that not only the timing, but also the shapes of these signals influence the synaptic modifications. The model is based on a Hebbian learning rule which correlates the NMDA synaptic conductance with the postsynaptic signal at synaptic location as the pre- and postsynaptic quantities. As compared to a previous paper (Saudargiene et al., 2004), here we show that this rule reproduces the generic STDP weight change curve by using real neuronal input signals and combinations of more than two (pre- and post-synaptic) spikes. We demonstrate that the shape of the STDP curve strongly depends on the shape of the depolarising membrane potentials, which induces learning. As these potentials vary at different locations of the dendritic tree, model predicts that synaptic changes are location-dependent. The model is extended to account for the patterns of more than two spikes of the pre- and postsynaptic cells. The results show that STDP weight change curve is also activity-dependent.

Keywords: Spike-timing-dependent plasticity; synaptic modifications; Hebbian learning; back-propagating spike; dendritic spike; neuronal activity.

\section{Introduction}

It is believed that learning and memory in biological neurons are based on the modifications of the synaptic strengths (Benett, 2000). Synapses are weakened or strengthened depending on the order and temporal difference of the pre- and postsynaptic activity. If the presynaptic spike precedes the postsynaptic spike $(T>$ $0)$, the synapse is potentiated, while it is depressed if the temporal order is reversed $(T<$ $0)$. This phenomenon is called spike-timingdependent-plasticity (Markram et al., 1997; Bi and Poo, 2001).

However, recent physiological experiments suggest that the properties of synaptic modifications may depend not only on timing, but also on the location of the synapse (Golding et al., 2002). i.e. synaptic strength is regulated by local learning rules. This assumption is supported by the fact the signals which drive synaptic plasticity strongly depend on the location at the neuron where a synapse is located (Häusser and Mel, 2003; Golding et al., 2002). Close to the soma back-propagating or, more distally, dendritic spikes provide the necessary post-synaptic depolarisation (Magee and Johnston, 1997; Golding et al., 2001, 2002; Larkum et al., 2001).

The natural activity of biological neurons is much more complex than that used in the experimental conditions of STDP induction. In these experiments the synaptic inputs are paired with postsynaptic action potentials (Magee and Johnston, 1997; Markram et al., 1997; Bi, 2002) or a synapse is stimulated with low or high frequency inputs (Bear, 1995). In natural conditions of learning multiple pre- and postsynaptic spikes may occur within milliseconds and bursting is observed (Paulsen and Sejnowski, 2000; Bi, 2002). How such neuronal activity influences synapse potentiation and depression still remains under debate (Bi, 2002). It has been argued that the spike pairs may cause independent effects (Kempter et al., 1999; Song et al., 2000; van Rossum et al., 2000) or interact with each other (Froemke and 
Dan, 2002; Eisele and Miller, 2002) such that the contribution of one given spike pair depends on the presence of other spikes. For example, it was observed that the first spike pair diminishes the influence of the following spike pair in layer 2 and 3 Pyramidal neurons (Froemke and Dan, 2002) implying that synaptic modifications are activitydependent.

In this study we present a biophysical model of STDP which can account for more realistic spiking patterns and capture the dependence of synaptic changes on the shapes of the pre- and postsynaptic signals. The model correlates the NMDA synaptic conductance and a postsynaptic signal, dependent on the postsynaptic depolarisation at the location of the synapse. The influence of the activity of the pre- and post-synaptic cells is incorporated in the model by introducing efficacies of each spike. In this study we use real neuronal signals and triplets of spikes as compared to another recent paper (Saudargiene et al., 2004) where the simulations were based on artificial signals and only spike pairs were considered. Also the learning rule differs. Here we will show that the new model reproduces the generic STDP weight change curve and is sensitive to the shapes of the postsynaptic membrane potential, responsible for learning induction. As this signal varies along the dendritic tree, the model predicts that STDP is location-dependent. We find that the most pronounced differences in synaptic modification rules should be in proximal and distal dendrites - anti-symmetrical STDP versus symmetrical weight change curve where only potentiation is observed, respectively. Moreover we note that STDP characteristics are influenced by the the spiking pattern of the pre- and post-synaptic cells.

\section{Biophysical model of STDP}

The biophysical model of STDP implements a dendritic compartment with a plastic synapse which consists of NMDA and AMPA channels (Fig. 1).

The AMPA channels are the ones that mainly express the plasticity it by changing the synaptic strength $\rho$ (Lisman, 1994). Our model is concerned with plasticity induction, therefore, no steps have been undertaken to explicitly model processes that modify AMPA channels.

The NMDA channels are essential for plasticity induction as their blockade to a large degree prevents STDP (Nishiyama et al., 2000; Golding et al., 2002). First, they serve as a coincidence detector between the pre- and postsynaptic activity (Markram et al., 1997). Second, open NMDA channels enable $\mathrm{Ca}^{2+}$ ion influx into the postsynaptic cell. It is believed that NMDA channel-mediated $\mathrm{Ca}^{2+}$ influx triggers complex chain reactions involving CaMKII, calmodulin, calcineurin and results in plasticity (Lisman, 1994; Bi, 2002).

In our model we associate the presynaptic signal with the time- function of the NMDA synaptic conductance given by:

$g(t)=\bar{g} c_{N}(t)=\bar{g} \frac{e^{-t / \tau_{1}}-e^{-t / \tau_{2}}}{1+\kappa e^{-\gamma V_{m}(t)}}$

where $\bar{g}_{N}=4 n S$ is a peak conductance, $\tau_{1}=$ $40 \mathrm{~ms}, \tau_{2}=0.33 \mathrm{~ms}$ are time constants, $V_{m}$ is the membrane potential, $\eta=0.33 / m M,\left[M^{2+}\right]=$ $1 m M, \gamma=0.06 / m V$ (Koch, 1999).

A strong depolarisation of the postsynaptic cell is necessary for learning induction (Golding et al., 2002). This depolarisation not only unblocks the NMDA channels, but also opens voltage-gated $\mathrm{Ca}^{2+}$ channels (Medina et al., 1999). The fraction $I_{S}$ of the total postsynaptic current $I(t)=$ $C \frac{d V_{m}(t)}{d t}$ may be associated with the $C a^{2+}$ influx, where $C$ is the membrane capacitance. We define this current $I_{S}$ as a postsynaptic quantity. It has a slower time course as the membrane potential $V_{m}$. We note that $\mathrm{Ca}^{2+}$ influx still occurs relatively fast but its elimination (for example via uptake mechanisms) is slow (Maravall et al., 2000). To capture this effect we filter the total current $I$ with the low pass filter $h$ :

$I_{S}=\varphi I(t) * h(t)=\varphi C\left(\frac{d V_{m}}{d t}\right) * h(t)$,

where $\varphi<1$ is the constant indicating that $\mathrm{Ca}^{2+}$ is a fraction of the total current, the asterisk denotes the convolution and filter function $h$ is expressed:

$h(t)=\sigma\left(e^{-t / \tau_{h 2}}-e^{-t \tau_{h 1}}\right)$, 
$\sigma$ is a scaling factor, $\tau_{h 1}=1 \mathrm{~ms}, \tau_{h 2}=40 \mathrm{~ms}$ are the filter parameters, chosen following (Maravall et al., 2000; Shouval et al., 2002). This filtering process is a major difference to our older study (Saudargiene et al., 2004), where only unfiltered signals were used.

The synaptic weight $\rho$ of the plastic synapse is updated according to a Hebbian learning rule which correlates the NMDA synaptic conductance with a postsynaptic current at the location of the synapse:

$$
\frac{d \rho}{d t}=\mu c_{N}(t) I_{S}(t)
$$

where $\mu$ is the learning rate, $c_{N}$ is the NMDA channel opening function, the pre-synaptic influence quantity, and $I_{S}$ is the current, the postsynaptic quantity.

The quantity $c_{N} I_{S}$ can be understood as the $\mathrm{Ca}^{2+}$ influx which is gated by a channel opening function $c_{N}$. Thus, the final weight change $\Delta \rho$, given by the integral, is determined by the $\mathrm{Ca}^{2+}$ concentration.

This final weight change $\Delta \rho$ after one pre-post pairing is expressed as $\left(T=t_{\text {post }}-t_{\text {pre }}\right)$ :

$$
\begin{array}{ll}
\Delta \rho(T)=\mu \int_{0}^{\infty} I_{S}(t-T) c_{N}(t) d t, & T \geq 0 \\
\Delta \rho(T)=\mu \int_{0}^{\infty} I_{S}(t) c_{N}(t+T) d t, & T<0
\end{array}
$$

The correlation is performed in a temporally causal way by applying the shift operation to either the current $I_{S}$ for $T \geq 0$ or to the NMDA channel function for $T<0$ in Eqs. 5, 6).

\section{STDP curves at different locations of the dendritic tree}

We applied our learning rule (Eq. 4) to model the synaptic changes using real depolarising signals, which trigger synaptic plasticity. These potentials vary along the dendritic tree. We use a short and steep BP action potential to obtain the synaptic changes close to the soma, and long shallow dendritic spike to account for synaptic modifications in the distal parts. The dendritic spike and BP-spike, measured $860 \mu \mathrm{m}$ and $210 \mu \mathrm{m}$ from the soma, respectively, are presented in Fig. 2 A, C) and have been adopted from (Larkum et al., 2001; Stuart et al., 1997). The depolarisation coming from these spikes is very strong, therefore we may neglect the contribution of the plastic synapse. We calculate the derivative of the membrane potential using the recorded shapes of spikes $V_{m}$, filter it with the filter $h$ to account for a slower $\mathrm{Ca}^{2+}$ dynamics and substitute the obtained current $I_{S}$ in the learning rule (Eq. 4). We do not model the processes of plasticity expression, therefore for the sake of simplicity we absorb the constant $\varphi$, membrane capacitance $C$ from Eq. 2 and the learning rate $\mu$ from Eq. 4 into a product $\mu \varphi C=1$ while using Eqs. 5, 6 . In principle, $\mu$ could be chosen to account for the amplification mechanisms which, however, were not meant to be implemented in this model.

The results are presented in Fig. $2 \mathrm{~B}$, D. We obtain an anti-symmetrical weight change curve, corresponding to differential Hebbian learning, if the depolarisation is provided by a steep backpropagating spike (Fig. $2 \mathrm{D})$. The synapse is weakened if $T<0$ and strengthened if $T>0$. However, we observe a curve more similar to Hebbian learning if the depolarisation comes from the shallow dendritic spike. The synaptic weight grows even for negative $T>-20 \mathrm{~ms}$ values. (Fig. 2 B). The shape of the depolarising potential strongly influences the STDP curve. Although the generic anti-symmetrical form of STDP is obtained in both cases, the transition from LTD to LTP is observed at different $T$ values. The shift towards a more Hebbian characteristic becomes stronger when rising flank of the depolarisation signal gets shallower (Saudargiene et al., 2004).

As the depolarising potentials vary in different parts of the dendritic tree, the results suggest that synaptic modifications depend on the location of the synapse and predict that proximal synapses are modified according to a differential Hebbian learning rule and distal synapses more according to a Hebbian learning rule, where LTD induction is prevented even for negative $T$ values $(T>-20 \mathrm{~ms}$ in the case presented). 


\section{Model extension for triplets of spikes}

So far we applied the model to estimate the synaptic modifications caused by one single preand postsynaptic spike pair. However, it was observed that the effect from the first pre- and postsynaptic spike pair dominates over the following spike pairs decreasing their influence (Froemke and Dan, 2002). In order to account for such suppression effects we modify the learning rule (Eq. 4) as follows:

$$
\frac{d \rho}{d t}=\mu c_{N}^{s u p}(t) I_{S}^{s u p}(t)
$$

where

$$
c_{N}^{\text {sup }}(t)=\sum_{i} \theta^{\text {pre }}\left(t_{i}^{\text {pre }}, t_{i-1}^{\text {pre }}\right) c_{N}\left(t-t_{i}^{\text {pre }}\right)
$$

and

$$
I_{s}^{\text {sup }}(t)=\sum_{i} \theta^{\text {post }}\left(t_{i}^{\text {post }}, t_{i-1}^{\text {post }}\right) I_{s}\left(t-t_{i}^{\text {post }}\right)
$$

The new quantities $c_{N}^{\text {sup }}$ and $I_{s}^{\text {sup implement }}$ the suppression of the pre- and postsynaptic activity by weighting the channel opening function $c_{N}$ and current $I_{s}$ with the efficacies $\theta^{\text {pre }}$ and $\theta^{\text {post }}$ of the $i^{t h}$ pre- and $i^{t h}$ postsynaptic spikes.

The efficacies $\theta^{\text {pre }}$ and $\theta^{\text {pre }}$ are expressed as:

$\theta^{\text {pre }}\left(t_{i}^{\text {pre }}, t_{i-1}^{\text {pre }}\right)=1-e^{\left(t_{i}^{\text {pre }}-t_{i-1}^{\text {pre }}\right) / \tau_{s}^{\text {pre }}}$,

and

$$
\theta^{\text {post }}\left(t_{i}^{\text {post }}, t_{i-1}^{\text {post }}\right)=1-e^{\left(t_{i}^{\text {post }}-t_{i-1}^{\text {post }}\right) / \tau_{s}^{\text {post }}} .
$$

where $t_{i}, t_{i-1}$ are the timing of the event $i$ and the preceding event $i-1$, parameters $\tau_{s}^{\text {pre }}$, $\tau_{s}^{\text {post }}$ are the suppression time constants of the pre- and postsynaptic activity, respectively. The terms $\theta^{\text {pre }}$ and $\theta^{\text {post }}$ define the efficacy of the $i^{\text {th }}$ event depending on the proximity of the preceding $(i-1)^{t h}$ event. Influence of the $i^{t h}$ event decreases if it follows the $(i-1)^{t h}$ event within a narrow time window. The approach for defining the efficacies of the spikes has been adopted from Froemke and Dan (2002).

We applied the modified learning rule Eq. 7 to model the synaptic changes induced by triplets of spikes. The third additional spike was introduced either pre- or postsynaptically, thus forming " $2 / 1$ " triplets (2 presynaptic spikes and 1 postsynaptic spike) or " $1 / 2$ " triplets (1 presynaptic spike and 2 postsynaptic spikes). As before, we investigated the influence of the location of the synapse by applying a short BP-spike and a shallow long dendritic spike as a source of depolarisation. The results are presented in Fig. 3.

We form a " $2 / 1$ " triplet by adding a presynaptic spike $S_{1}^{\text {pre }}$ (bold) after a postsynaptic spike with a temporal difference $T_{1}=-20 \mathrm{~ms}$ and keep $T_{1}$ constant (Fig. 3, A). Another presynaptic spike $S_{2}^{\text {pre }}$ arrives at varying temporal differences $T$ in respect to the postsynaptic spike. Three different temporal situations of these spikes are shown. 1) For $T<-20 m s$ the STDP curve is dominated by the influence of the closest presynaptic spike $S_{1}^{\text {pre }}$ which occurs always at a fixed time $T_{1}=-20 \mathrm{~ms}$ and suppresses the influence of $S_{2}^{\text {pre }}$. 2) As soon as $S_{2}^{\text {pre }}$ arrives earlier than $S_{1}^{p r e}$, the situation reverses, $S_{2}^{p r e}$ diminishes the effect of $S_{1}^{\text {pre }}$ and the weight change curve starts largely depending on the $S_{1}^{p r e}$ arrival time, i.e. on the temporal difference $T$. 3) A transition from depression to potentiation is observed when $T$ becomes positive and the depolarisation is provided by a BP-spike (Fig. $3 \mathrm{~A}$, solid). In addition, for large positive $T$ the influence of $S_{2}^{\text {pre }}$ becomes small and $S_{1}^{\text {pre }}$ starts dominating which even leads to depression. The location of the synapse also has an important role. Depression is very pronounced if the source of depolarisation is a steep BP-spike which corresponds to proximal dendritic parts (Fig. 3 A, solid). Only potentiation is observed if plasticity is driven by dendritic spikes which occur in distal parts (Fig. $3 \mathrm{~A}$, dotted). In principle, similar results are obtained if spike $S_{1}^{\text {pre }}$ occurs with $T_{1}=-40 \mathrm{~ms}$. (Fig. $3, \mathrm{C}$ ). If $T_{1}>0$, we obtain mainly potentiation as the spike $S_{1}^{\text {pre }}$ arrives before the postsynaptic spike.

Similar simulations were carried out for triplets " $1 / 2$ " formed by introducing an additional postsynaptic spike $S_{1}^{\text {post }}$ (bold) at $T_{1}=20 \mathrm{~ms}$ after a presynaptic spike (Fig. 3, B). Again, three temporal situations are presented. 1-2) Spike $S_{2}^{\text {post }}$ suppresses the contribution of $S_{1}^{\text {post }}$ as long as it arrives earlier than $S_{1}^{\text {post }}$, i.e. $T<20 \mathrm{~ms}$. However, for very large negative $T$ the $S_{1}^{\text {post }}$ takes over and results even in potentiation, if the depolarisation is provided by a BP-spike (Fig. $3 \mathrm{~B}$, solid). 3) As 
soon as $S_{2}^{\text {post }}$ occurs later than $S_{1}^{\text {post }}(T>20 \mathrm{~ms})$, it's influence is suppressed by the preceding spike $S_{1}^{\text {post }}$. As before, we observe the pronounced differences between synaptic changes close and far away from the soma. For a short BP-spike (proximal location) potentiation is obtained, if $T$ has large negative values $T<-40 \mathrm{~ms}$ (Fig. $3 \mathrm{~B}$, solid). For a shallow dendritic spike (distal location) we get potentiation if $T>-40 \mathrm{~ms}$ (Fig. 3 $\mathrm{B}$, dotted). These two cases clearly differ from the commonly observed STDP. The similar results for a case $T_{1}=40 \mathrm{~ms}$ are presented in Fig. 3 D. Negative $T_{1}$ leads to the depression, as the spike $S_{1}^{\text {post }}$ precedes the presynaptic spike. The obtained results match the physiological observations of Froemke and Dan (2002).

\section{Discussion}

The typical approach to model STDP is to assume a certain weight change curve which does not depend on the local properties of the cell, e.g. (Gerstner et al., 1996; Abbott and Song, 1999; Song et al., 2000). A few more detailed models take into consideration the postsynaptic signal which is associated with the membrane potential, e.g. (Rao and Sejnowski, 2001; Karmarkar and Buonomano, 2002; Shouval et al., 2002) and observe that its shape influences the shape of the weight change curve. In our previous work we presented a biophysical model of STDP and showed that the weight change curve strongly depended on the shapes of the depolarising membrane potential (Saudargiene et al., 2004).

Here in the new study we investigated the influence of the realistic depolarising membrane potentials on STDP. In addition, we extended the model to account for the influence of the spiking pattern of the pre- and postsynaptic cells. The results show that the properties of the STDP weight change curve are influenced by the location of the synapse as the strong depolarisation, necessary for plasticity induction, changes its shape along the dendrite and is provided by different mechanisms such as BP-spikes close to the soma and dendritic spikes in the distal dendritic parts. Therefore we predict that rules of synaptic modifications are location-dependent. Close to the soma the synaptic modifications are bidirectional, described by an anti-symmetrical STDP curve. In the distal parts synapses undergo potentiation even for negative values of $T$. The same learning rule leads to different synaptic modifications and it is self-adjusting following the shapes of the depolarisation source in different locations of the dendritic tree.

The notion that site specific plasticity can exist in real neural networks is currently indirectly supported by findings showing that the membrane properties of real neurons strongly change along its dendrites (Stuart and Häusser, 2001; Häusser et al., 2000). Recent physiological experiments also confirm the predictions of our rule about transition from temporally anti-symmetric to symmetric weight change curve (Kampa and Stuart, 2003). Three postsynaptic spikes, hence a more prolonged dendritic signal, were used to drive plasticity and resulted in LTP for negative values of $T$.

The model was modified to account for the synaptic modifications induced not only by a single spike pair, but also by multiple pre- and postsynaptic spikes. Specifically, we have modeled synaptic changes for triplets of spikes. The obtained results matched physiological observations which show that the contribution of the given spike pair is suppressed by the preceding preor postsynaptic spikes (Froemke and Dan, 2002). This suppression effect was simulated by introducing the spike efficacies in Eq. 7 and in this way implementing short-term depression which occurs presynaptically (Wu and Borst, 1999; Wong et al., 2003) and postsynaptically (Lisman, 1994; $\mathrm{Bi}, 2002)$. In principle, it is possible to incorporate advanced models of short-term plasticity, e.g.(Giugliano et al., 1999; Scheuss et al., 2002). Our model could be applied to find synaptic modifications induced by bursting and natural spike trains (Paulsen and Sejnowski, 2000; Froemke and Dan, 2002).

It is known that postsynaptic signals strongly depend both on the activity and the electrotonic distance from the soma. BP-spikes are attenuated during repetitive somatic firing at constant high frequency and fail to invade distal dendritic parts (Williams and Stuart, 2000; Colbert 
et al., 1997). However, high-frequency physiological spike trains occurring within short temporal periods propagate back into the dendritic tree and induce dendritic spikes (Williams and Stuart, 2000). Our learning rule not only accounts for the activity pattern, but also for the shapes of the depolarising potentials and in this way combines the effects of activity and location on STDP.

Location- and activity-dependent learning rules can provide the neurons with a substantially diversified set of computational properties which has the potential to enhance the computational power of such networks tremendously.

\section{References}

Abbott, L. S., Song, S., 1999. Temporal asymmetric Hebbian learning, spike timing and neuronal response variability. In: Kearns, M. S., Solla, S., Cohn, D. A. (Eds.), Advances in Neural Information Processing Systems. Vol. 11. MIT Press, Cambridge, MA, pp. 69-75.

Bear, M., 1995. Mechanism for a sliding synaptic modification threshold. Neuron 15, 1-4.

Benett, M. R., 2000. The concept of long term potentiation of transmission at synapses. Prog. Neurobiol. 60, 109-137.

Bi, G. Q., 2002. Spatiotemporal specificity of synaptic plasticity: cellular rules and mechanisms. Biol. Cybern. 87, 319-332.

Bi, G.-Q., Poo, M., 2001. Synaptic modification by correlated activity: Hebb's postulate revisited. Annu. Rev. Neurosci. 24, 139-166.

Colbert, C. M., Magee, J. C., Hoffman, D., Johnston, D., 1997. Slow recovery from inactivation of Na channels underlies the activity-dependent attenuation of dendritic action potentials in hippocampal ca1 pyramidal neurons. J. Neurosci. 17 (17), 6512-6521.

Eisele, M., Miller, K., 2002. Hidden Markov Model of cortical synaptic plasticity: derivation of the learning rule. Advances in Neural Information Processing Systems 15, 253-260.
Froemke, R., Dan, Y., 2002. Spike-timingdependent synaptic modification induced by natural spike trains. Nature 416, 433-8.

Gerstner, W., Kempter, R., van Hemmen, J. L., Wagner, H., 1996. A neuronal learning rule for sub-millisecond temporal coding. Nature 383, 76-78.

Giugliano, M., Bove, M., Grattarola, M., 1999. Fast calculation of short-term depressing synaptic conductances. Neural Comput. 11, 1413-26.

Golding, N., Kath, W. L., Spruston, N., 2001. Dichotomy of action-potential backpropagation in ca1 pyramidal neuron dendrites. J. Neurophysiol. 86, 2998-3010.

Golding, N. L., Staff, P. N., Spurston, N., 2002. Dendritic spikes as a mechanism for cooperative long-term potentiation. Nature 418, 326331.

Häusser, M., Mel, B., 2003. Dendrites: bug or feature? Current Opinion Neurobiol. 13, 372383.

Häusser, M., Spruston, N., Stuart, G. J., 2000. Diversity and dynamics of dendritic signaling. Science 11, 739-744.

Kampa, B., Stuart, G., 2003. Dendritic mechanisms involved in spike-timing-dependent plasticity. Proceedings of Sixth IBRO World Congress on Neuroscience, 2003, Prague, Czech Republic, 331 .

Karmarkar, U. R., Buonomano, D. V., 2002. A model of spike-timing dependent plasticity: One or two coincidence detectors? J. Neurophysiol. 88, 507-513.

Kempter, R., Gerstner, W., van Hemmen, J., 1999. Hebbian learning and spiking neurons. Phys. Rev. 59, 4498-4514.

Koch, C., 1999. Biophysics of Computation. Oxford University Press. 
Larkum, M. E., Zhu, J. J., Sakmann, B., 2001. Dendritic mechanisms underlying the coupling of the dendritic with the axonal action potential initiation zone of adult rat layer 5 pyramidal neurons. J. Physiol. (Lond.) 533, 447-466.

Lisman, J., 1994. The CaM kinase II hypothesis for the storage of synaptic memory. Trends Neurosci. 17, 406-412.

Magee, J. C., Johnston, D., 1997. A synaptically controlled, associative signal for Hebbian plasticity in hippocampal neurons. Science 275, 209-213.

Maravall, M., Mainen, Z., Sabatini, B., Svoboda, K., 2000. Estimating intracellular calcium concentrations and buffering without wavelength ratioing. Biophys. J. 78, 2655-2667.

Markram, H., Lübke, J., Frotscher, M., Sakmann, B., 1997. Regulation of synaptic efficacy by coincidence of postsynaptic APs and EPSPs. Science $275,213-215$.

Medina, I., Leinekugel, X., Ben-Ari, Y., 1999. Calcium-dependent inactivation of the monosynaptic NMDA EPSCs in rat hippocampal neurons in culture. Eur. J. Neurosci. 11, 2422-30.

Nishiyama, M., Hong, K., Mikoshiba, K., Poo, M., Kato, K., 2000. Calcium stores regulate the polarity and input specificity of synaptic modification. Nature 408, 584-588.

Paulsen, O., Sejnowski, T. J., 2000. Natural patterns of activity and long-term synaptic plasticity. Current Opinion Neurobiol. 10, 172-179.

Rao, R. P. N., Sejnowski, T. J., 2001. Spiketiming-dependent Hebbian plasticity as temporal difference learning. Neural Comp. 13, 22212237.

Saudargiene, A., Porr, B., , Wörgötter, F., 2004. How the shape of pre- and postsynaptic signals can influence stdp: A biophysical model. Neural Comp. .

Scheuss, V., Schneggenburger, R., Neher, E., 2002. Separation of presynaptic and postsynaptic contributions to depression by covariance analysis of successive EPSCs at the Calyx of Held Synapse. J. Neurosci. 22, 728-739.

Shouval, H. Z., Bear, M. F., Cooper, L. N., 2002. A unified model of NMDA receptor-dependent bidirectional synaptic plasticity. Proc. Natl. Acad. Sci. (USA) 99 (16), 10831-10836.

Song, S., Miller, K. D., Abbott, L. F., 2000. Competitive Hebbian Learning through spiketiming-dependent synaptic plasticity. Nature Neurosci. 3, 919-926.

Stuart, G., Spruston, N., Sakmann, B., Häusser, M., 1997. Action potential initiation and backpropagation in neurons of the mammalian central nervous system. Trends Neurosci. 20, 125131.

Stuart, G. J., Häusser, M., 2001. Dendritic coincidence detection of EPSP and action potentials. Nature Neurosci. 4 (1), 63-79.

van Rossum, M., Bi, G., Turrigiano, G., 2000. Stable hebbian learning from spike timingdependent plasticity. J. Neurosci. 20, 8812-21.

Williams, S., Stuart, G., 2000. Backpropagation of physiological spike trains in neocortical pyramidal neurons: Implications for temporal coding in dendrites. J. Neurosci. 20, 8238-8246.

Wong, A., B.P., G., Billups, B., Forsythe, I., 2003. Distinguishing between presynaptic and postsynaptic mechanisms of short-term depression during action potential trains. J Neurosci. $23,4868-77$.

Wu, L., Borst, J., 1999. The reduced release probability of releasable vesicles during recovery from short-term synaptic depression. Neuron $23,821-32$. 


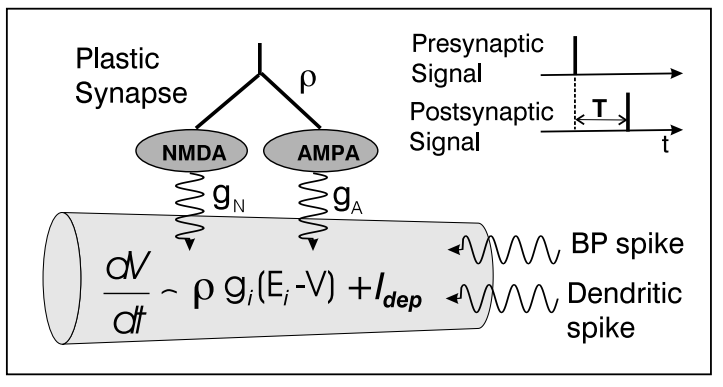

Figure 1. Schematic diagram of the biophysical model. A plastic synapse consists of NMDA and AMPA channels and has the strength $\rho$. The source of depolarisation which removes $M^{2+}$ block from the NMDA channel may take the form of a BP- or dendritic spike.

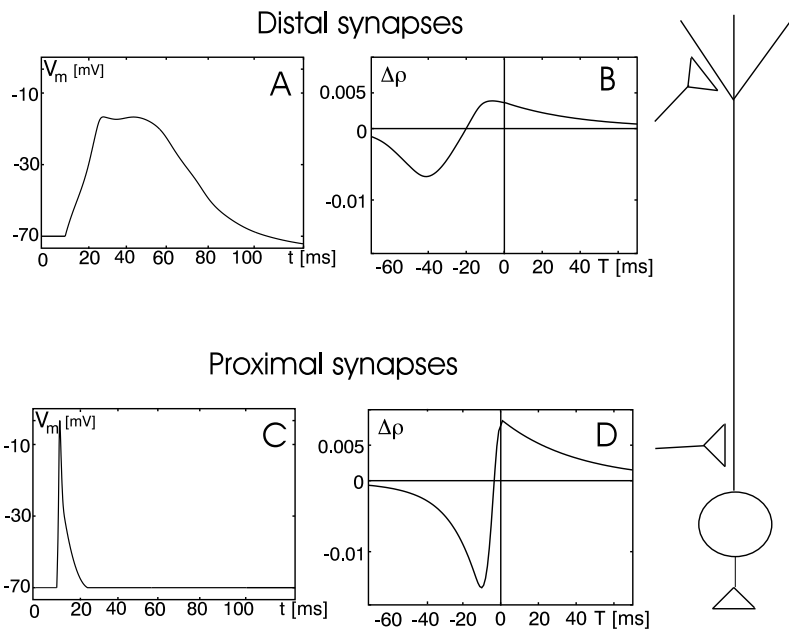

Figure 2. Location-dependent STDP curves. A) In distal parts slow and wide local dendritic spikes drives the plasticity (measured $860 \mu \mathrm{m}$ from the soma, adopted from Larkum et al. (2001)). B) The resulting weight change curve. Potentiation is observed even for negative values $T>-20 \mathrm{~ms}$. C) Close to the soma short BP-spikes induce plasticity (measured $210 \mu \mathrm{m}$ from the soma, adopted from Stuart et al. (1997)). D) The obtained weight change curve is anti-symmetrical. Scale factors $\sigma$ in Eq. 3: B) $\sigma=0.0256$, D) $\sigma=0.0373$. 

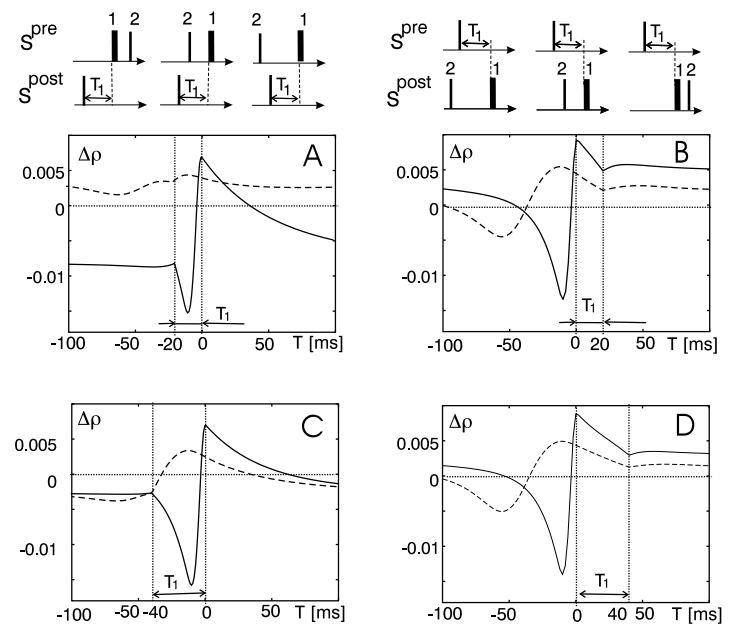

Figure 3. Activity-dependent STDP curves for A,C) " $2 / 1$ " triplets, B,D) " $1 / 2$ " triplets. Source of depolarisation: BP- (solid) and dendritic spike (dotted) (Fig. 2 C,A). A,C) The presynaptic spike $S_{1}^{\text {pre }}$ (bold) occurs $T_{1}=-20 \mathrm{~ms}$ or $T_{1}=$ $-40 \mathrm{~ms}$ after the postsynaptic spike. The temporal interval $T$ between the other presynaptic spike $S_{2}^{p r e}$ and the postsynaptic spike varies. B,D) The postsynaptic spike $S_{1}^{\text {post }}$ (bold) occurs $T_{1}=20 \mathrm{~ms}$ or $T_{1}=40 \mathrm{~ms}$ after the presynaptic spike. The temporal interval $T$ between the other postsynaptic spike $S_{2}^{\text {post }}$ and the presynaptic spike varies. $\tau_{s}^{\text {pre }}=\tau_{s}^{\text {post }}=0.1 \mathrm{~s}$ in Eqs. 10, 11. 